\title{
Hallervorden Spatz disease (Pantothenate Kinase associated Neurodegeneration): a rare case report
}

\author{
Gupta $A^{1}$, Soujanya B. $S^{1}$, Shetty $S^{2}$ \\ ${ }^{1}$ Dr. Ankur Gupta, ${ }^{1}$ Dr. Soujanya B. S. Post Graduate resident in Department of Pediatrics, ${ }^{2}$ Dr. Sumanth Shetty, \\ Professor in Department of Pediatrics; all authors are affiliated to K.S. Hegde Medical Academy, Nitte University, \\ Mangalore, Karnataka, India.
}

Address for Correspondence: Dr. Ankur Gupta, Department of Paediatrics, K.S. Hegde Medical Academy, Mangalore, Karnataka. Email:drankurgupta1987@gmail.com

\begin{abstract}
Hallervorden Spatz disease (HSD), is an autosomal recessive disorder characterised by regression of attained milestones, mental retardation and movement disability. We present a case of 6 year old male child admitted with regression of attained milestones, mainly language, ataxia and extrapyramidal symptoms since 2 years. MRI brain with contrast had shown typical "eye of the tiger" sign. Child was symptomatically improved with Tab. Trihexyphenidyl. Reporting such cases would benefit us to be familiarised with this rare disease and to differentiate it from other static and progressive neurological illnesses.
\end{abstract}

Key words: Hallervorden spatz disease, Ataxia, Eye of the tiger

\section{Introduction}

Hallervorden Spatz disease (HSD), now considered as pantothenate kinase associated neurodegeneration (PKAN) is a rarely seen autosomal recessive neurodegenerative disorder characterised by progressive degenerative changes in basal ganglia, globus pallidus and reticular part of the substantia nigra [1]. Clinical presentations include dystonia, dysarthria, dementia, severe mental retardation and ataxia with rare features as rigidity, choreoathetosis, seizures, optic atrophy and pigmentary retinopathy [2]. The characteristic MRI brain pattern in T2 weighted image is "eye of the tiger" sign. Only six cases have been reported from India, clinical diagnosis based on clinical and MRI characteristics [3-6]. We report a clinically and radiologically proven case of Hallervorden Spatz disease from southern India.

\section{Case Report}

A 6 year old male child born to a second degree consanguinous married couple came with presenting complaints of difficulty in walking and repeated falls since 2 years duration, which has become more in frequency in past 6 months. Child also had difficulty in seeing far objects, more in night. History of regression of developmental milestones especially language is present. There was history of developmental delay mainly in gross and fine motor skills. On handling child used to get dystonic posturing of mainly upper limb and face. Also there is dysarthria which is more since last 6 months. Child had two other normal siblings. On examination: multiple atrophic and healing scars present over the face and scalp. CNS examination showed features of dystonia and dysarthria with normal reflexes. In view of neurodegenerative disorder mainly involving grey matter, MRI scan of head with contrast was done which had shown b/l symmetrical T2/FLAIR hyperintensity in globus pallidus showing peripheral blooming on susceptibility weighted sequences suggestive of 'Eye of tiger sign' [Figure-1]. EEG was reported to be normal. Neurology opinion suggested neurodegeneration with brain iron accumulation [NBIA] disorder. Ophthalmological evaluation shown severe astigmatism with myopic features and was advised corrective measures for the same. Child was discharged on Tab.

Manuscript received: $20^{\text {th }}$ September 2016

Reviewed: $4^{\text {th }}$ October 2016

Author Corrected; $16^{\text {th }}$ October 2016

Accepted for Publication: $30^{\text {th }}$ October 2016 
Trihexphenidyl with regular follow up visits. On follow up child have shown marked improvement in features of dystonia and gait difficulty.

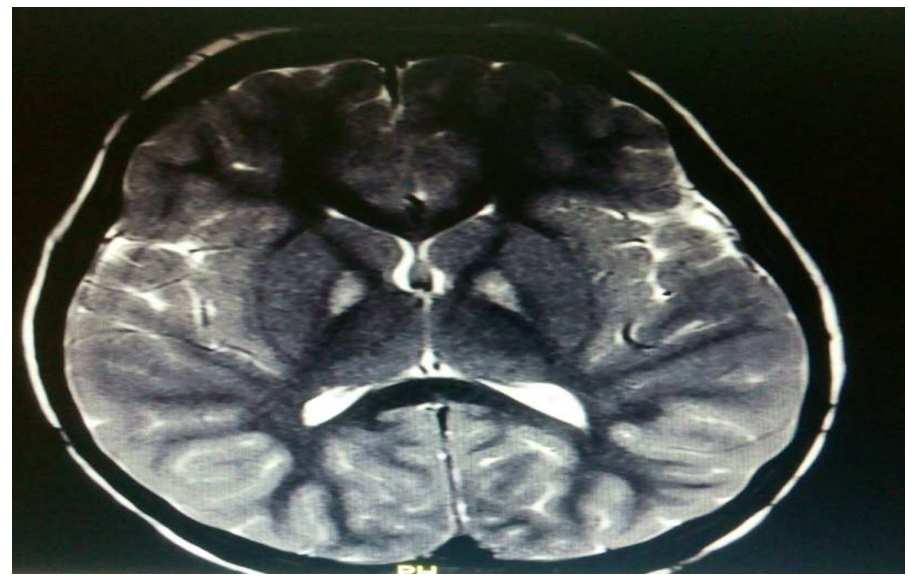

Figure-1: MRI head with contrast showing B/L symmetrical T2/FLAIR hyperintensity in globus pallidus showing peripheral blooming suggestive of 'Eye of tiger sign'.

\section{Discussion}

Hallorverden Spatz disease is a rare neurodegenerative disorder. The term Neurodegeneration with brain iron accumulation type- 1 (NBIA-1) has been recently used in publications [7]. It was 1st described by Hallervorden and Spatz in 1922 and characterised by familial brain degeneration with iron accumulation [8]. Defect in pantothenate kinase2 producing gene located in chromosome 20p13-p12.3 is seen in most of the cases, so also known as Pantothenate kinase-associated neurodegeneration (PKAN) [9]. There are two types of NBIA as early onset and late onset as described in Figure: 2 [9].

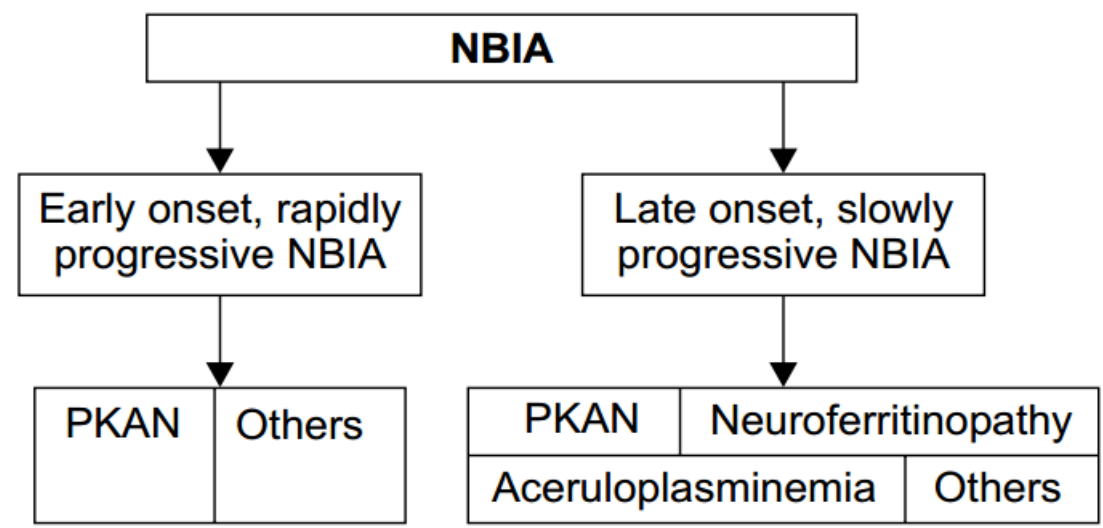

Figure-2: Classification of NBIA [9]

Pathological findings in brain include rusty brown pigmentation due to iron deposition, axonal swelling and neuronal loss with gliosis mostly in the globus pallidus and pars reticularis of substantia nigra [10].

The exact etiology of HSD is unknown. One accepted hypothesis is that abnormal peroxidation of lipofuscin to neuromelanin and deficient cysteine deoxygenase causes abnormal iron accumulation in the brain. While portions of the globus pallidus and pars reticularis part of substantia nigra have high iron content in healthy individuals.

HSD is relentlessly progressive. The symptom most commonly appears in late childhood or early adolescence between Age of 7 and 15 years. This occurs in all races and has a similar frequency in both genders [1]. The disease progression is 
characterized by progressive dementia, corticospinal signs (spasticity, hyer reflexia) and extrapyramidal signs. Seizures have been noticed in few patients. Affected individuals typically die in the second or third decade. Based on various clinical features, the following criteria have been given (Table- 1) [11].

Table-1: Diagnostic features of Hallervorden spatz disease [11].

\begin{tabular}{|c|c|c|}
\hline Obligate Features & Collaborate Features & Exclusion Features \\
\hline $\begin{array}{c}\text { Onset during first two } \\
\text { decades }\end{array}$ & Pyramidal tract signs \\
disease & Progressive cognitive impairment & $\begin{array}{c}\text { Family history of Huntington's disease, } \\
\text { caudate atrophy or autosomal dominant } \\
\text { movement disorder }\end{array}$ \\
\hline $\begin{array}{c}\text { Progressive nature of the } \\
\text { Extra-pyramidal dysfunction }\end{array}$ & Retinitis pigmentosa & Absence of extrapyramidal signs \\
\hline & Positive family history & Abnormal ceruloplasmin or copper level \\
\hline & $\begin{array}{c}\text { Hypodensity in basal ganglia on } \\
\text { MRI brain }\end{array}$ & \\
\hline & $\begin{array}{c}\text { Abnormal cytoplasm in } \\
\text { lymphocytes }\end{array}$ & \\
\hline & Sea blue histocytes on bone marrow & \\
\hline
\end{tabular}

The eye-of-the-tiger sign on the MRI brain and clinical findings has correlated to a diagnosis of HSD in this child. The characteristic MRI findings are symmetrical hyperintense signal changes in the anterior medial globus pallidus with peripheral hypointensity in the globus pallidus on bilateral sides on T2 - weighted images characterized as "eye of the tiger" pattern [12]

Management is mainly symptomatic. Drugs advised for spasticity and dystonia are baclofen and trihexphenidyl. Other therapies for relieving dystonia are intramuscular botulinum toxin, intrathecal baclofen, stereotactic pallidotomy, bilateral thalamotomy and deep brain stimulation [13]. A brain permeable iron chelator, VK-28 which provides Neuroprotection by the inhibiting both basal and Fe/ascorbate induced mitochondrial membrane lipid peroxidation, has shown considerable results in rats. Its efficacy is comparable to desferal, a proteolytic iron chelator [14].

The differential diagnosis includes neuroacanthocytosis, wilson disease, juvenile ceroid lipofuscinosis, huntington disease. Other differential diagnosis of iron deposition in the basal ganglia with "eye-of-the-tiger" sign includes aceruloplasminemia and neuroferritinopathy. These are definite conditions of abnormal iron metabolism but unlike Hallervorden-spatz disease they present in adult or late life [10].

\section{Conclusion}

The treatment of children with Hallervorden Spatz disease (HSD) remains mainly symptomatic. A multidisciplinary team approach which involves physical, occupational and speech therapists is required to improve functional skills and quality of life.

Funding: Nil, Conflict of interest: Nil

Permission from IRB: Yes

\section{References}

1. Hayflick SJ, Westaway SK, Levinson B, Zhou B, Johnson MA, Ching KH, Gitschier J. Genetic, clinical, and radiographic delineation of Hallervorden-Spatz syndrome. N Engl J Med. 2003;348:33-40.
2. Hartig MB, Hortnagel K, Garavaglia B, Zorzi G, Kmiec T, Klopstock T, Rostasy K, Svetel M, Kostic VS, Schuelke M, Botz E, Weindl A, Novakovic I, Nardocci N, Prokisch H, Meitinger T. Genotypic and phenotypic spectrum of PANK2 mutations in patients with neurodegeneration with brain iron accumulation. Ann Neurol. 2006; 59(2):248-56.

3. Kaushik A, Longia S, Jagadeesh R, Kishore V. Hallervorden-spatz disease. Indian Pediatr 1995; 32: 483-5.

4. Singhi PD, Mitra S. Hallervorden spatz disease: Late infantile type. J Child Neurol 1997;12:281-2. 
5. Shah J, Patkar D, Patankar T, Krishnan A, Prasad S, Limdi J. Hallervorden spatz disease: MR imaging. J Postgrad Med 1999;45:114-7.

6. Rao C, Murthy V, Hedge R, Asha, Vishwanath. Hallervorden spatz disease. Indian J Pediatr 2003; 70:513-14.

7. Neumann M, Adler S, Schulter D, et al. Alphasynuclein accumulation in a case of neurodegeneration with brain iron accumulation type 1 (NBIA-1, formely hallervorden-spatz syndrome) with widespread cortical and brainstem-type lewy bodies. Acta Neuropathol (Berl) 2000; 100 (5); 568-574.

8. Gregory A, Hayflick SJ. Folia Neurodegeneration with brain iron accumulation. Neuropathology 2005; 43:286- 96.

9. Schneider SA, Hardy J, Bhatia K. Iron Accumulation in Syndromes of Neurodegeneration with Brain Iron Ac cumulation 1 and 2 - causative or consequential?. J Neurol Neurosurg Psychiatry. 2009 (Jan); 80 (6); 589-90.
10. M.C.Sharma, N. Aggarwal, M. Bihari, V. Goyal, S. Gaikwed, S. Vaishya, C. Sarkar Hallervorden Spatz disease: $\mathrm{MR}$ and pathological findings of a rare case Neurology India March 2005 Vol 53 Issue 1:102-104.

11. Swaiman KF. Hallervorden - Spatz syndrome and brain iron metabolism. Arch Neurology 1991; 48:12851293.

12. Sethi KD, Adams RJ, Loring DW, el Gammal T. Hallervorden-Spatz syndrome: Clinical and magnetic resonance imaging correlations: Ann Neurol. 1988;24: 692-94.

13. Castelnau P, Cif L, Valente EM, Vayssiere N, Hemm S, Gannau A, Digiorgio A, Coubes P. Pallidal stimulation improves pantothenate kinase-associated neurodegeneration. Ann Neurol. 2005; 57:738-741.

14. Shachar DB, Kahana N, Kampel V, Warshawsky A, Youdim MB. Neuroprotection by a novel brain permeable iron chelator, VK-28, against 6- hydrodopamine lesion in rats. Neuropharmacology 2004;46: 254-63.

\section{How to cite this article?}

Gupta A, Soujanya B. S, Shetty S. Hallervorden Spatz disease (Pantothenate Kinase associated Neurodegeneration): a rare case report. Int. J PediatrRes.2016;3(10):759-762.doi:10.17511/ijpr.2016.i10.08 